\section{Gemeinsam die Zukunft gestalten}

Unter diesem Motto ging vor wenigen Wochen der 3. gemeinsame Deutsche Kongress für Orthopädie und Unfallchirurgie in Berlin zu Ende.

Die Veranstaltung ging über 4 Tage, 13 Parallelsitzungen sorgten für einige Verwirrung, aber auch dafür, dass jeder Teilnehmer etwas für ihn Interessantes finden konnte. Beeindruckend war wieder die hohe Präsenz der Veranstaltungen zum Thema „Obere Extremität“. Insgesamt 11 wissenschaftliche Sitzungen, 2 Expertenrunden, 2 Kurse, 3 Veranstaltungen unter dem Motto „Tipps und Tricks" und 2 Kommissionssitzungen beschäftigten sich mit aktuellen Themen aus dem Bereich "Schulter - Ellenbogen - Hand“.

Das Schultergelenk stand wieder im Fokus - über kein anderes Gelenk wurden in Berlin so viele Vorträge zu Verletzungen und Erkrankungen, Diagnostik, Operation und Rehabilitation gehalten und diskutiert.

Nebenbei tagten die großen Fachgesellschaften, DGOOC und DGU - die Gründung einer gemeinsamen „Deutschen Gesellschaft für Orthopädie und Unfallchirurgie (DGOU)“ steht ja bekanntermaßen unmittelbar bevor.

Unter dem Dach dieser neuen umfassenden Fachgesellschaft sollen die vielen einzelnen Sektionen, Arbeitskreise, Arbeitsgemeinschaften etc. der "alten“ Gesellschaften zusammengeführt werden.

Dies ist ein sinnvolles Ziel - der Weg dorthin wird aber nicht einfach sein, da die korrespondierenden wissenschaftlichen Gruppierungen selbstverständlich auf unterschiedlichen, über die Jahre gewachsenen organisatorischen und personellen Strukturen aufbauen und nicht immer die gleichen Schwerpunkte und Ziele verfolgen. Nicht wenige AG's und AK's treibt die Sorge um, in den neuen Strukturen unterzugehen.

Dies gilt, wie für alle Bereiche, natürlich auch für die Gruppierungen der Schulter-, Ellenbogen- und Handchirurgie. Es ist sinnvoll und wichtig, dass hier bald eine gemeinsame Basis gefunden wird um die vorhandenen wertvollen wissenschaftlichen und berufspolitischen Aktivitäten zu bündeln und in eine gemeinsame Richtung zu lenken.

Die Deutsche Vereinigung für Schulter- und Ellenbogenchirurgie (DVSE e.V.) als eigenständige Vereinigung einerseits, andererseits aber auch als Sektion der DGOOC, hat den gemeinsamen Weg für Orthopäden und Unfallchirurgen von Anfang an beschritten.

Etwa $40 \%$ der 270 Mitglieder sind originäre Unfallchirurgen, die übrigen $60 \%$ sind Orthopäden. Unter den Neumitgliedern verschiebt sich das Gewicht mehr und mehr auf die Seite der Unfallchirurgen, wobei mit dem neuen Facharzt in Kürze diese Grenzen endlich vollständig aufgehoben sein werden.

Die letzten 4 Jahrestagungen wurden im Wechsel von Unfallchirurgen und Orthopäden ausgerichtet, die Hauptthemen der Kongresse verteilten und verteilen sich auch in Zukunft über beide Schwerpunkte.

Der Vorstand der DVSE ist paritätisch besetzt, der letzte Präsident war ein Unfallchirurg und im nächsten Frühjahr stehen mit offenem Ergebnis Vorstandswahlen ins Haus.

Dies ist ein Grund mehr alle in der DGU aktiven Gruppierungen, die sich mit der arthroskopischen oder offenen Chirurgie der Schulter als Arbeitsgemeinschaften oder Arbeitskreise beschäftigen, mit offenen Armen in unsere gemeinsame Vereinigung aufzunehmen.

Das Motto wird lauten: Gemeinsam die Zukunft gestalten.

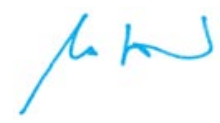

Ihr

Markus Loew 